\title{
A Distributed Scheduling Algorithm Based on Minimal Distortion for Video Stream in Wireless Networks
}

\author{
LIN Nan \\ College of Software Technology, Zheng Zhou University, Henan Zhengzhou, 450002, China
}

Keywords: Video distortion, Distributed scheduling, Optimal solution, Transmission delay rate, Distortion rate

\begin{abstract}
A video stream distributed scheduling algorithm with minimal distortion in wireless network is propose in this paper. The algorithm adopts the novel model to capture the video distortion, establish video distortion model, and further modify the queuing model with $\mathrm{M} / \mathrm{G} / 1$ so as to get the video streaming distortion and the delay distribution correlation function. In addition, the network congestion delay constraint for the system is also optimized to reduce the distortion rate of video stream. In order to minimize the optimal solution, the routing and packet allocation problems are considered to get the minimum routing congestion and reduce the network transmission delays. Comparative analysis of experimental data and simulation results show that our proposed algorithm has achieved good results and performance in reducing the distortion rate of video stream, shortening transmission delay rate and controlling the packet loss rate.
\end{abstract}

\section{Introduction}

In the network video service, Researcher [1] proposed a high efficient scheduling algorithm for VBR-encoded video stream with zero delay, where one of the key techniques is to use storage and network resources efficiently. Several zero-delay stream scheduling algorithms are introduced, and the advantages and disadvantages of each scheduling methods are analyzed. ZDB-VBR algorithm is proposed to solve the waiting delay and reduce the consumption of server bandwidth maximally. Researchers [2, 3, 4] proposed communication scheduling application algorithm. According to the transmission char-control data transmission and Mock stream's constraints, this algorithm can ensure the stability of EPA network and can satisfy the fluency of block stream. This algorithm[5] defines video stream as "block stream", meanwhile gives its mathematical model, and considers block stream as period so as to divide the same blocks. Researchers [6] proposed a scheduling framework for adaptive video delivery over cellular networks, which is a network resource management framework.

The scheduling of video stream in the multi channel network with multiple wireless interfaces is more complex. Compared to the traditional data communication, in wireless multi hop networks the questions which should be focused on for the proposed algorithm in this paper includes: How to allocate the channel to make the link to achieve normal data transmission without interference; How to allocate the appropriate data transmission rate for a given channel and link; How to minimize the distortion of video stream in the chosen channel and link; How to adopt a fair principle to make the multiuse video streams transmitted simultaneously. The questions above are very important for the performance of the algorithm, involving the MAC and application layers of a network, which is also a challenging cross-layer-control problem.

\section{Video stream minimal distortion function}

It is assumed that there are $N=\left(c_{1}, c_{2}, \cdots, c_{n}\right)$ nodes and $L=\left(l_{1}, l_{2}, \cdots, l_{s}\right)$ links in the multi channel network with multiple wireless interfaces, $f$ represents non overlapping channels and that there are $m$ network interfaces on each node. In order to take the channel diversification into account, we assumed that $v_{i}$ represents the data transmission rate of the link $l_{i}$ in the channel $f_{i}$. There are $U=\left(u_{1}, u_{2}, \cdots, u_{k}\right)$ users in the network. Each user can connect the source nodes and destination 
nodes through the router, and each user's traffic can be routed to multiple alternate routes. We use $H_{u}^{l}$ to represent the routing matrix, when the user uses the link $l$, then $H_{u}^{l}=1$, otherwise $H_{u}^{l}=0$; $M(u)$ represents the number of optional routing routes of the user $u ; Q_{k}(u)$ represents portion of the video stream on the link $k$ routed from user $u ; L_{c}$ represents the link set which are chosen to transmit data in channel $f_{i}$. For the link $l$, we can obtain the link balance rate $\xi(l)$ :

$$
\xi(l)=\kappa \frac{Y_{\text {in }}(l)}{Y_{\text {out }}(l)}
$$

Where $Y_{\text {in }}(l)$ denotes the link input function; $Y_{\text {out }}(l)$ represents the link output function; $\kappa$ represents a constant.

The calculation formula for input function $Y_{\text {in }}(l)$ is as following:

$Y_{\text {in }}(l)=\sum_{l=1}^{L_{c}} H_{u}^{l} \sum_{u=1}^{U} v(u) Q_{k}(u)$

Where $v(u)$ represents the video stream transmission rate of the user $u$.

The calculation formula for output function $Y_{\text {out }}(l)$ is as following:

$$
Y_{\text {out }}(l)=\sum_{l \in f} \log _{2}\left(Y_{\text {in }}(l)\right)
$$

It is assumed that each radio can only be tuned to one channel. For the successful communication from the link $l$ in the channel $f_{i}$, then both the source node and the destination node must tune a radio to channel $f_{i}$. For all the data packets traversing this link, the average delay is determined by the blocking of the wireless link, we use the classical M/G/1 queuing model for analysis, there is a link between the transmission capacity $E(l)$ and average delay, it can be written as follows:

$E(l)=\frac{Y_{\text {out }}(l)}{(\xi(l)-\theta)}$

Where $\theta$ denotes the variance caused by excessive allocation.

For the user $u$, the average delay on the link $l$ is as following:

$D_{l}=\sum_{l=1}^{L} \frac{\varepsilon((\xi(l)-\theta))}{\pi Y_{\text {out }}(l)} H_{u}^{l}$

Where $\varepsilon$ represents the average packet size.

For user $u$, the total packet loss rate on link $l$ is as following:

$P_{\text {loss }}=\frac{\overline{P_{\text {loss }}}}{T_{h}}+\frac{\left(1-\overline{P_{\text {loss }}}\right) P\left(\text { delay }>T_{h}\right)}{D_{l}}$

Then, the total additional distortion caused by the packet loss $S_{\text {loss }}$ is as following:

$S_{\text {loss }}=\chi \pi P_{\text {loss }}=\chi \pi \frac{\overline{P_{\text {loss }}}}{T_{h}}+\chi \pi \frac{\left(1-\overline{P_{\text {loss }}}\right) P\left(\text { delay }>T_{h}\right)}{D_{l}}$

\section{Distributed scheduling algorithm with minimal distortion}

We use multi path router for multiple potential path to reduce the total system congestion contained in each user. Inspired by [7], we take into consideration that each user video stream increment enters into the channel $f_{i}$, which contains $k$ links. We assumed that the link capacity is known, and on the 
$\operatorname{link} l_{u}^{k}$ the congestion reaches the minimum. The average delay on each link is inversely proportional to $E(l)$, the congestion for the video stream increment $W_{u}$ on the link $l_{u}^{k}$ is as following:

$\psi=\sum_{l \in L} \frac{Y_{\text {out }}(l)+W_{u}}{E(l)-W_{u}}$

We take the congestion optimization of the whole network into consideration; The formula can be transformed into:

$\psi^{\prime}=\sum_{u=1}^{U} \sum_{l=1}^{L}\left(\frac{Y_{\text {out }}(l)+W_{u}}{E(l)-W_{u}}-\frac{Y_{\text {out }}(l)}{E(l)}\right)=\min \left\{\sum_{u=1}^{U} \sum_{l=1}^{L} \frac{W_{u}}{E(l)}\right\}$

By finding the link $l_{u}^{k}$ between the source and destination nodes, we achieved the minimizing congestion in the network, and then the optimization problem can be changed into:

$\min _{l_{u}^{k}}\left\{\sum_{u=1}^{U} \sum_{l=1}^{L} \frac{W_{u}}{E(l)}\right\}$

We took into consideration both the problem of routing and rate allocation. The sufficient and necessary conditions for the optimization of the network congestion minimization are that routing and rate allocation ratio for user video stream from the network must meet the requirements of the video stream transmission, which makes the ratios of the distortion $S_{c}$ caused by source compression and the additional distortion $S_{\text {loss }}$ caused by packet loss to the video stream increment $W_{u}$ reaching the maximum respectively. i.e.

$$
\frac{d S_{c}}{d W_{u}}=-\frac{\sqrt{\pi \theta_{1}}}{\left(W_{u}-W_{u}^{\prime}\right)^{2}}
$$

\section{Simulation and analysis of experiments}

The equipments used in this paper and the related parameters configuration are as follows: The bit rate of the video codec we used is $1000 \mathrm{Mbps}$; A standard video sequence with a length of 200 frame is used as the encoding files [8]; The length of the group of pictures is 4 frames and the frame rate is 35fps; The macro-cycle is $25 \mathrm{~ms}$ and the network bandwidth is $120 \mathrm{M}$; The delay jitter is $5 \mathrm{~ms}$; The maximum and minimum delay of the pictures frame is $20 \mathrm{~ms}$ and $10 \mathrm{~ms}$ respectively. The source equipment for the video data stream is a network camera of full frame rate $25 \mathrm{fbps}$. The intra-frame coding model used by the camera provides the video data stream. The simulation tool used in the experiment is QualNet, and the simulation of the video decoding and transmission protocol is in QualNet; the operation computer is equipped with Intel core i5-2400, 4G ram and processor clocked at $3.1 \mathrm{GHz}$.

In order to verify the efficiency of the distributed scheduling algorithm with minimal distortion in the reduction of the video stream data distortion, in the experiments, we gradually increased the video streams data, and obtained the ratio of the distorted video stream data to the total data, and finally we got the Figure 1 results. As the data distribution shows in Figure 1, The video stream distortion ratio in the algorithm proposed in this paper is the smallest, only at $23.4 \%$ even when the video data streams number reached 800 , while in the algorithm proposed by Jiasi Chen and Kai-lung Hua, the ratio is more than $30 \%$. Obviously, our algorithm proposed in this paper achieves better efficiency in the control of video distortion. That is because in the algorithm, we used the M/G/1 queuing model to analyze the video distortion factors and used the distributed scheduling algorithm with minimal distortion to solve them. 


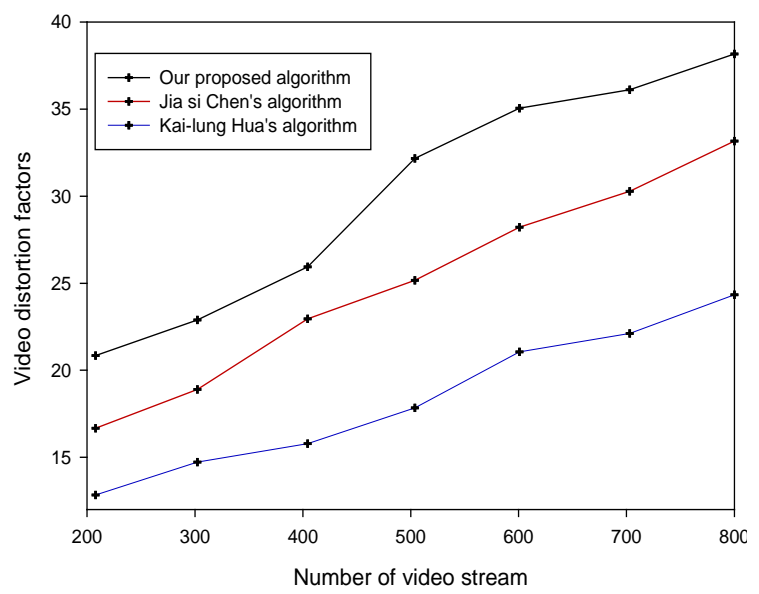

Figure 1 The video stream distortion rate

The network delay is the main factor that causes the video stream distortion. When an ordered set of video stream data fails to transmit within the valid time interval due to transmission delays, it will cause the video distortion. Then, it is very important to control the network delay. In order to verify the efficiency of the distributed scheduling algorithm with minimal distortion in the controlling of the network delay, in the experiments simulation, we gradually increased the video streams data, and obtained the network delay time of the video stream transmission, and finally we got the Figure 2 results. As the data distribution shows in Figure 2, With the increase of video streams number, the network delay time gradually increased, but the increasing speed slows down gradually. In the algorithm proposed in this paper, when the video streams number reaches 800, the network delay time is $13.5 \mathrm{~ms}$, while in both the algorithms proposed by Jia si Chen and Kai-lung Hua, the network delay time is more than $17 \mathrm{~ms}$. Thus, the algorithm proposed in this paper achieves better efficiency in the control of network delay time of the video stream transmission. That is because in the algorithm, we took the influence of network delay on the video stream distortion into consideration and chose the optimal route links to minimize the congestion in the network, and then reduced network delay.

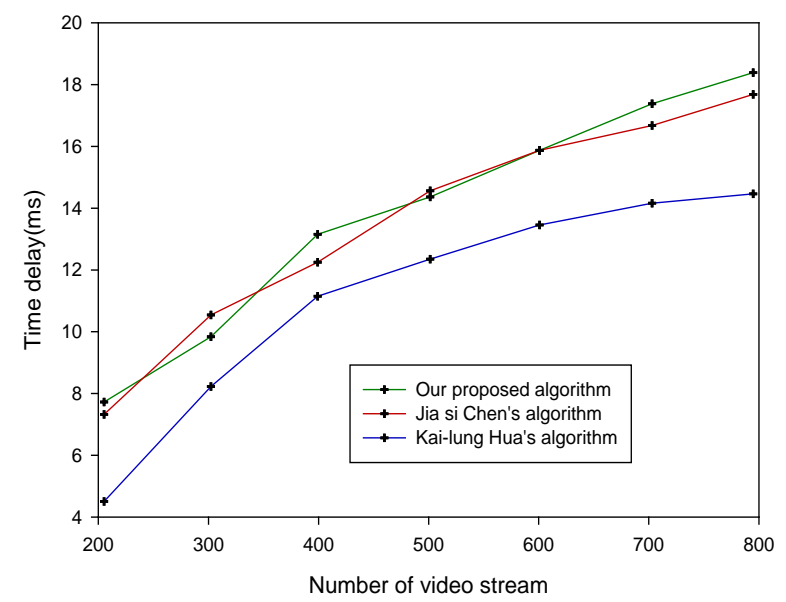

Figure 2 The network delay time of video stream transmission

Figure 3 shows the packet loss rate when the video packet length is increased. The higher the network packet loss rate is, the more serious the video stream data loss situation is. In the algorithm proposed in this paper, the routing and the allocation of the data transmitting rate were taken into consideration, as well as the optimization of the whole network congestion and the delay constraint of the system. Therefore the video packet loss was controlled, and during all the experiments simulation, the packed loss rate is less than $14 \%$, with average at $9.4 \%$. while in both the algorithms proposed by Jiasi Chen and Kai-lung Hua, the average packet loss rate is $19.8 \%$ and $17.1 \%$, respectively. According to the data results from the experiments, the packed loss rate is smaller in the algorithm proposed in this paper. 


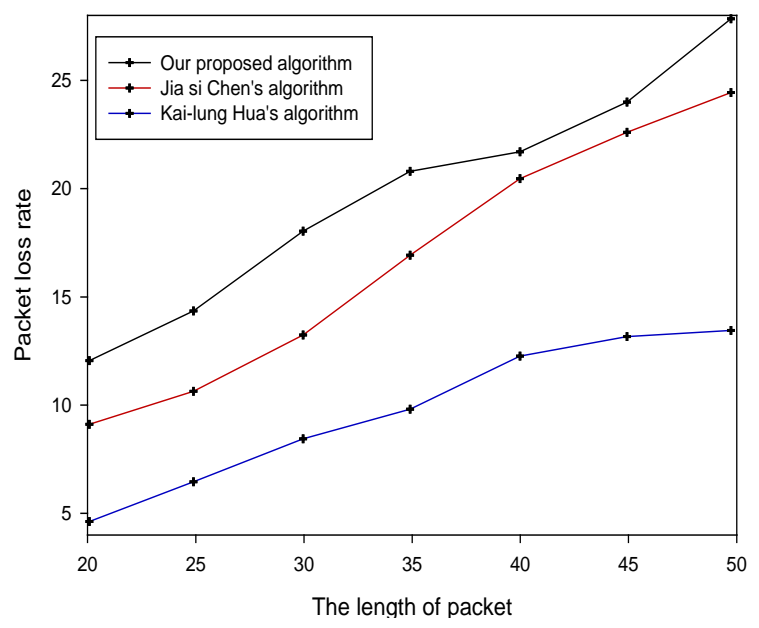

Figure 3. The network packet loss rate

\section{Conclusion}

In the video communication industry with huge amount of data, in order to get video data with higher quality at the terminals and to improve the utilization of the network, the video stream scheduling of distortion has become a focus of research in wireless network. This paper proposes a video stream distributed scheduling algorithm with minimal distortion in wireless network. In this algorithm, we obtained video stream minimal distortion function. And by taking the links balance rate and the delay constraint on the links, as well as the optimization of routing choice on the multiple routes, into consideration, we took the video stream distortion rate and the network delay time of the data transmission under control. According to the experimental simulation and comparative analysis, by the video stream distributed scheduling algorithm with minimal distortion in wireless network, we can achieve better efficiency in reducing the video stream distortion, controlling the network delay time of the video stream transmission and reducing the network packet loss rate.

\section{References}

[1] Chen W, Cao J, Wan Y. QoS-aware virtual machine scheduling for video streaming services in multi-cloud[J]. Tsinghua Science and Technology, 2013, 18(3): 308-317.

[2] Ashraf A, Jokhio F, Deneke T, et al. Stream-based admission control and scheduling for video transcoding in cloud computing[C]//Cluster, Cloud and Grid Computing (CCGrid), 2013 13th IEEE/ACM International Symposium on. IEEE, 2013: 482-489.

[3] Baboescu, Florin, Sumeet Singh, and George Varghese. "Packet classification for core routers: Is there an alternative to CAMs?." INFOCOM 2003. Twenty-Second Annual Joint Conference of the IEEE Computer and Communications. IEEE Societies. Vol. 1. IEEE, 2003.

[4] Taylor, David E., and Jonathan S. Turner. "Classbench: A packet classification benchmark." INFOCOM 2005. 24th Annual Joint Conference of the IEEE Computer and Communications Societies. Proceedings IEEE. Vol. 3. IEEE, 2005.

[5] Taylor, David E., and Jonathan S. Turner. "Scalable packet classification using distributed crossproducing of field labels." INFOCOM 2005. 24th Annual Joint Conference of the IEEE Computer and Communications Societies. Proceedings IEEE. Vol. 1. IEEE, 2005.

[6] Warkhede, Priyank, Subhash Suri, and George Varghese. "Fast packet classification for two-dimensional conflict-free filters." INFOCOM 2001. Twentieth Annual Joint Conference of the IEEE Computer and Communications Societies. Proceedings. IEEE. Vol. 3. IEEE, 2001. 
[7] Chen J, Mahindra R, Khojastepour M A, et al. A scheduling framework for adaptive video delivery over cellular networks [C]// Proceedings of the 19th annual international conference on Mobile computing \& networking. ACM, 2013: 389-400.

[8] Bhatia R, Lakshman T V, Netravali A, et al. Improving mobile video streaming with link aware scheduling and client caches[C] //INFOCOM, 2014 Proceedings. IEEE, 2014: 100-108. 